\title{
Vacuum spacetimes with toroidal null infinities
}

\author{
Bernd G Schmidt $\dagger$ \\ Max-Planck-Institut für Gravitationsphysik, Albert-Einstein-Institut, Schlaatzweg 1, 14473
}

Potsdam, Germany

Received 18 March 1996

Abstract. It is shown that some known solutions of Einstein's vacuum field equations admit null infinities such that the sections of the null hypersurface at infinity are toroidal.

PACS numbers: 0420G, 0420H, 0430

\section{Introduction}

The conformal field equations developed by Friedrich [1] seem to be an ideal tool to perform numerical calculations of an asymptotically flat spacetime on a finite grid. This was sucessfully tested by Hübner [2] for the case of the spherical collapse of a scalar field. The next step is to do a $(2+1)$-dimensional calculation, for example an axisymmetric one. For this purpose one needs initial data to begin the evolution, and exact solutions to check the code. Due to the well known difficulties at spacelike infinity, one presently has to use Cauchy hypersurfaces which intersect future null infinity scri ${ }^{+}$. Such Cauchy surfaces are called hyperboloidal. In the special case where the second fundamental form is proportional to the metric of the initial hypersurface, the momentum constraint just demands that the mean curvature is constant. The Hamiltonian constraint can be formulated as a semilinear elliptic equation. Such data are analogous to time symmetric data in the usual formulation of the Cauchy problem.

In [3], such initial data are constructed which have an evolution admitting smooth null infinity [4]. The basic result is as follows. Choose any smooth Riemannian metric $h$ on some orientable, compact 3-manifold $M=\tilde{M} \cup \partial M$ with boundary and a function $\rho$ which defines the boundary by $\rho=0, \mathrm{~d} \rho \neq 0$. Suppose that the trace-free part of the second fundamental form of the boundary of $\tilde{M}$ vanishes. Then there exists a unique solution $u$ of the Hamiltonian constraint ( $R$ is the Ricci scalar of $u^{4} \rho^{-2} h$ )

$$
R\left(u^{4} \rho^{-2} h\right)=-6,
$$

which has a smooth extension to the boundary of $\tilde{M}$ and $\left(\tilde{M}, \tilde{g}=u^{4} \rho^{-2} h, \tilde{\chi}=\right.$ constant $\left.\tilde{g}\right)$ is a solution of the constraints in spacetime.

Let me consider some examples:

(1) Suppose $M$ is the closed unit ball in $R^{3}$ with its standard metric. Since the solution $u$ is unique the initial data must be spherically symmetric and turn out to describe a hyperboloid in Minkowski space.

$\dagger$ E-mail address: bernd@aei-potsdam.mpg.de 
(2) $M=S^{2} \times[-1,1]$ with its standard metric. Again the initial data are spherically symmetric. This case defines a hypersurface in the Kruskal spacetime intersecting both future null infinities.

(3) $M=S^{1} \times S^{1} \times[-1,1]$. The solution evolving from this initial data set must have two null infinities with toroidal sections. The initial data admit two commuting Killing vectors. One can show in addition that there is a local group of isotropies. The generalized Birkhoff theorem tells us that these data must evolve into a part of the analogue of the Schwarzschild solution with planar symmetry. This is the solution A3 in the classification of Ehlers and Kundt [5], which had already been found by Levi-Civita in 1916. This solution was independently rediscovered several times and appears also as a Kasner solution or a Robinson-Trautman solution. It is a 'cosmological solution' admitting null infinity.

This reasoning tells us that there is a well known solution which can be used to check the code for the conformal field equations. Particularly nice is that the Killing vectors have no fixed points. So there are no axis problems and one can fully concentrate on the study of the radiation at infinity.

The possibility of toroidal null infinity was first mentioned by Newman and Unti in [12]. The asymptotic symmetry groups of null infinities with non-spherical sections were investigated by Foster [13]. As far as I am aware, explicit examples are given the first time in this paper.

The plan of the paper is as follows. In section 2, I calculate null infinities for the A3 solution. Section 3 contains generalizations and describes solutions with just two commuting Killing vectors admitting null infinities with toroidal sections. It is amusing that there is still another class of such spacetimes with a global structure different from cylindrical or boost-rotational symmetry.

\section{Null infinity of $\mathbf{A 3}$}

The analogue of the Schwarzschild metric with planar symmetry is the metric A3 in the classification of Ehlers and Kundt:

$$
g=-\frac{1}{R} \mathrm{~d} T^{2}+R \mathrm{~d} R^{2}+R^{2}\left(\mathrm{~d} x^{2}+\mathrm{d} y^{2}\right) .
$$

It is defined for all real $x, y, T$ and $R \neq 0$. The isometry group is four dimensional. For $R>0$ the metric is static and for $R<0$ we have an Abelian three-parameter group of isometries, hence a Kasner solution. The obvious coordinate transformation reveals $\left(-\frac{1}{3}, \frac{2}{3}, \frac{2}{3}\right)$ for the Kasner exponents. $R=0$ is a curvature singularity because $R^{-3}$ is an eigenvalue of the Weyl tensor.

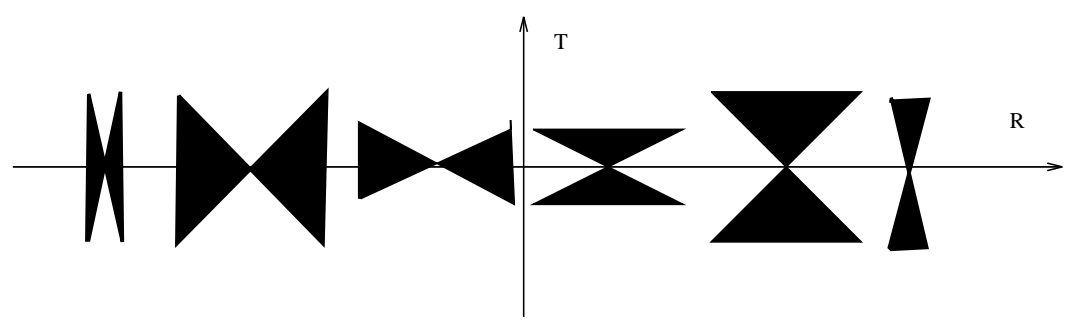

Figure 1. 
Figure 1 shows the structure of the null cones in the $R-T$ plane. (Black indicates the timelike directions.) In these coordinates the null lines are the parabolas $T=$ $\pm \frac{1}{2} R^{2}+$ constant. We expect null infinity in the directions of the null lines to infinity. Hence, only for the case $R<0$ we have the possibility to connect two scris by a spacelike hypersurface.

To demonstrate the existence of a conformal infinity we introduce a null coordinate $u$ by the transformations

$$
T=\frac{1}{2} R^{2}+u ; \quad \mathrm{d} T=R \mathrm{~d} R+\mathrm{d} u .
$$

The metric becomes

$$
g=-2 \mathrm{~d} R \mathrm{~d} u-\frac{1}{R} \mathrm{~d} u^{2}+R^{2}\left(\mathrm{~d} x^{2}+\mathrm{d} y^{2}\right)
$$

In the coordinate

$$
\bar{R}=\frac{1}{R}
$$

the metric is

$$
g=\frac{2 \mathrm{~d} \bar{R}}{\bar{R}^{2}} \mathrm{~d} u-\bar{R} \mathrm{~d} u^{2}+\frac{1}{\bar{R}^{2}}\left(\mathrm{~d} x^{2}+\mathrm{d} y^{2}\right) .
$$

Rescaling with a conformal factor $\Omega=\bar{R}$ one obtains the metric

$$
\bar{g}=\Omega^{2} g=2 \mathrm{~d} \bar{R} \mathrm{~d} u-\bar{R}^{3} \mathrm{~d} u^{2}+\left(\mathrm{d} x^{2}+\mathrm{d} y^{2}\right)
$$

which obviously allows a regular extension through the null hypersurface $\bar{R}=0$. The conformal extension is possible in both cases, $\bar{R}<0, \bar{R}>0$.

It is also instructive to consider the conformal extensions in double null coordinates. Using a second null coordinate $v$,

$$
T=-\frac{1}{2} R^{2}+v, \quad \mathrm{~d} T=-R \mathrm{~d} R+\mathrm{d} v, \quad R^{2}=v-u,
$$

we obtain

$$
g=-\frac{\mathrm{d} u \mathrm{~d} v}{ \pm \sqrt{|v-u|}}+(v-u)\left(\mathrm{d} x^{2}+\mathrm{d} y^{2}\right) .
$$

The two signs correspond to the cases $\bar{R}<0, \bar{R}>0$.

We expect null infinities for $u=$ constant, $v \rightarrow \infty$ and $v=$ constant, $u \rightarrow-\infty$ because $v-u>0$. With

$$
\bar{v}^{2}=\frac{1}{v}, \quad v>0 \quad \text { and } \quad \bar{u}^{2}=-\frac{1}{u}, \quad u<0
$$

we obtain

$$
g=-\frac{\mathrm{d} \bar{u} \mathrm{~d} \bar{v}}{ \pm \bar{v}^{2} \bar{u}^{2} \sqrt{\bar{u}^{2}+\bar{v}^{2}}}+\frac{\bar{u}^{2}+\bar{v}^{2}}{\bar{u}^{2} \bar{v}^{2}}\left(\mathrm{~d} x^{2}+\mathrm{d} y^{2}\right) .
$$

The conformal factor $\Omega=\bar{u} \bar{v}$ defines the rescaled metric

$$
\bar{g}=\Omega^{2} g=-\frac{\mathrm{d} \bar{u} \mathrm{~d} \bar{v}}{ \pm \sqrt{\bar{u}^{2}+\bar{v}^{2}}}+\left(\bar{u}^{2}+\bar{v}^{2}\right)\left(\mathrm{d} x^{2}+\mathrm{d} y^{2}\right)
$$

which is regular for $\bar{v}=0, \bar{u} \neq 0$ and $\bar{u}=0, \bar{v} \neq 0$.

Any hypersurface $\bar{v}=-\bar{u}+$ constant is spacelike in the case $R<0$ and intersects both scris.

To relate to the toroidal case treated in the introduction we only have to take the above metrics with some identification $x \bmod (a), y \bmod (b)$ for any two positive numbers $a, b$. 
We have thus demonstrated that the A3 solution has two null infinities which have toroidal sections if we do the appropriate identification.

There are more solutions with null infinities in the class of degenerate static vacuum solutions: for example, the solution A2 which has an isometry group $G_{1} \times G_{3}$. The $G_{3}$-orbits are hyperboloids of constant negative curvature. This solution can be used to construct null infinities whose sections are spheres with any finite number of handles. Details will be given elsewhere.

\section{Generalizations}

The solution discussed in the previous section has a four-parameter group of isometries like the Schwarzschild solution. In this section I construct solutions which are locally EinsteinRosen waves with two spacelike, hypersurface orthogonal Killing vectors. Globally, however, they differ from the cases investigated up to now: cylindrical waves [6], boostrotational spacetimes [7] and Gowdy universes [8].

We take the vacuum field equations for the metric

$$
g=\mathrm{e}^{2 M}\left(-\mathrm{d} t^{2}+\mathrm{d} z^{2}\right)+t\left(\mathrm{e}^{W} \mathrm{~d} x^{2}+\mathrm{e}^{-W} \mathrm{~d} y^{2}\right)
$$

from Isenberg and Moncrief [9]:

$$
\begin{aligned}
& W_{, t t}+\frac{1}{t} W_{, t}-W_{, z z}=0 \\
& M_{, t}=-\frac{1}{4 t}+\frac{t}{4}\left(\left(W_{, t}\right)^{2}+\left(W_{, z}\right)^{2}\right) \\
& M_{, z}=\frac{t}{2} W_{, t} W_{, z} .
\end{aligned}
$$

The integrability condition of the two equations (3.3) and (3.4) is the field equation (3.2), a Euler-Poisson-Darboux equation with index $\mu=\frac{1}{2}$. The corresponding Green function is known in terms of hypergeometric functions.

Consider first the case $W=0$. Integration of (3.3) gives $\mathrm{e}^{2 M}=t^{-\frac{1}{2}}$ and therefore the metric

$$
g=\frac{1}{\sqrt{t}}\left(-\mathrm{d} t^{2}+\mathrm{d} z^{2}\right)+t\left(\mathrm{~d} x^{2}+\mathrm{d} y^{2}\right) .
$$

This metric is isometric (up to a constant factor) to the metric A3 in the form given in (2.8). An obvious transformation of the $t$-coordinate would result in the Kasner form.

Are there solutions of (3.2) defining spacetimes with conformal infinities analogous to the A3 spacetime? I give two examples.

The function

$$
W=\frac{1}{\sqrt{t^{2}-z^{2}}}
$$

solves (3.2). Integration of (3.3), (3.4) gives

$$
2 M=-\frac{1}{2} \ln t-\frac{1}{4} \frac{t^{2}}{(t-z)^{2}(t+z)^{2}} .
$$

Knowing that $\mathrm{A} 3$ admits scri it is easy to check that this is also the case for this spacetime. Writing

$$
2 M=-\frac{1}{2} \ln t+N
$$


the metric is

$$
g=\frac{1}{\sqrt{t}} \mathrm{e}^{N}\left(-\mathrm{d} t^{2}+\mathrm{d} z^{2}\right)+t\left(\mathrm{e}^{W} \mathrm{~d} x^{2}+\mathrm{e}^{-W} \mathrm{~d} y^{2}\right) .
$$

Transforming to double-null coordinates

$$
2 u=t+z, \quad 2 v=t-z, \quad t=u+v,
$$

the metric becomes

$$
g=\frac{1}{\sqrt{(u+v)}} \mathrm{e}^{N}(-4 \mathrm{~d} u \mathrm{~d} v)+(u+v)\left(\mathrm{e}^{W} \mathrm{~d} x^{2}+\mathrm{e}^{-W} \mathrm{~d} y^{2}\right)
$$

and we can proceed as in section 2 to find the null infinity:

$$
\begin{aligned}
& \bar{u}^{2}=\frac{1}{u}, \quad \bar{v}^{2}=\frac{1}{v} \\
& g=-4 \frac{\mathrm{d} \bar{u} \mathrm{~d} \bar{v}}{\bar{v}^{2} \bar{u}^{2} \sqrt{\bar{v}^{2}+\bar{u}^{2}}} \mathrm{e}^{N}+\frac{\bar{v}^{2}+\bar{u}^{2}}{\bar{u}^{2} \bar{v}^{2}}\left(\mathrm{e}^{W} \mathrm{~d} x^{2}+\mathrm{e}^{-W} \mathrm{~d} y^{2}\right) .
\end{aligned}
$$

The conformal factor $\Omega=\bar{u} \bar{v}$ defines the rescaled metric

$$
\bar{g}=\Omega^{2} g=-4 \frac{\mathrm{d} \bar{u} \mathrm{~d} \bar{v}}{\sqrt{\bar{v}^{2}+\bar{u}^{2}}} \mathrm{e}^{N}+\left(\bar{v}^{2}+\bar{u}^{2}\right)\left(\mathrm{e}^{W} \mathrm{~d} x^{2}+\mathrm{e}^{-W} \mathrm{~d} y^{2}\right) .
$$

Since

$$
W=\frac{1}{2} \bar{u} \bar{v}, \quad N=-\frac{1}{64}\left(\bar{u}^{2}+\bar{v}^{2}\right)^{2}+\frac{1}{2} \ln 2
$$

are analytic at $\bar{v}=0$ and $\bar{u}=0$ we have two analytic null infinities. This spacetime is only defined for $t>z$.

The next, more complicated example has the same domain of definition as the solution $\mathrm{A} 3, t>0$. It is the analogue of the 'Weber-Wheeler-Bonnor' pulse [10,11]. It is determined by the following solutions of (3.2):

$$
W=\sqrt{2} C \sqrt{\frac{\sqrt{\left(a^{2}+t^{2}-z^{2}\right)^{2}+4 a^{2} z^{2}}+a^{2}+t^{2}-z^{2}}{\left(a^{2}+t^{2}-z^{2}\right)^{2}+4 a^{2} z^{2}}} .
$$

(For $a=0$ we obtain (3.6) up to a constant.) Integration of (3.3) gives

$$
\begin{gathered}
2 M=-\frac{1}{2} \ln t+\frac{1}{4} C^{2}\left(\frac{1}{a^{2}}-2 \frac{t^{2}\left(\left(a^{2}+t^{2}-z^{2}\right)^{2}-4 a^{2} z^{2}\right)}{\left(\left(a^{2}+t^{2}-z^{2}\right)^{2}+4 a^{2} z^{2}\right)^{2}}\right. \\
\left.+\frac{t^{2}-a^{2}-z^{2}}{a^{2} \sqrt{\left(a^{2}+t^{2}-z^{2}\right)^{2}+4 a^{2} z^{2}}}\right) .
\end{gathered}
$$

The same coordinate transformation and rescaling as above shows that we again obtain two analytic null infinities.

It is natural to ask which solutions of the wave equation (3.2) will define spacetimes with null infinities. I conjecture that it should be sufficient that the solution $W$ has a smooth extension to the null infinity defined by the solution A3. This has to be checked by an analysis of the field equations as was done in the boost-rotational case [7]. 


\section{References}

[1] Friedrich H 1981 Proc. R. Soc. A 378

[2] Hübner P 1996 Phys. Rev. D 53

[3] Andersson L, Chrusćiel P T and Friedrich H 1992 Commun. Math. Phys. 149

[4] Friedrich H 1983 Commun. Math. Phys. 91

[5] Ehlers J and Kundt W 1962 Gravitation ed Witten (New York: Wiley)

[6] Stachel J 1966 J. Math. Phys. 7

[7] Bičák J and Schmidt B G 1989 Phys. Rev. D 40

[8] Gowdy R H 1974 Ann. Phys. 83

[9] Isenberg J and Moncrief V 1990 Ann. Phys. 199

[10] Weber J and Wheeler J 1985 Rev. Mod. Phys. 29

[11] Bonnor W B 1957 J. Math. Mech. 6

[12] Newman E T and Unti T W J 1962 J. Math. Phys. 3

[13] Foster J 1987 Int. J. Theor. Phys. 26 